\title{
PAPER
}

\section{Angiotensin converting enzyme insertion/deletion genotype is associated with leukoaraiosis in lacunar syndromes}

\author{
A Hassan, A Lansbury, A J Catto, A Guthrie, J Spencer, C Craven, P J Grant, \\ J M Bamford
}

See end of article for authors' affiliations

.....................

Correspondence to: Dr J M Bamford, Department of Neurology, St James's University Hospital, Beckett Street, Leeds LS9 7TF, UK;

j.m.bamford@leeds.ac.uk

Received 19 April 2001 In revised form 19 September 2001

Accepted 20 September 2001

\begin{abstract}
Objectives: Pathological and clinical data suggest that patients presenting with ischaemic lacunar syndromes may be a heterogenous group. Those with isolated lacunar infarction are thought to have localised atherosclerosis whereas in those with coexisting leukoaraiois a distinct diffuse small vessel vasculopathy may be the predominant underlying pathology. The ACE insertion/deletion (I/D) polymorphism is an important candidate gene in ischaemic cerebrovascular disease but, where lacunar stroke specifically has been examined, there have been discrepant reports concerning a possible association. It was hypothesised that the influence of the ACE gene may be different among the two subgroups of ischaemic lacunar stroke reflecting the heterogeneity of the small vessel disease phenotype.

Methods: Eighty four consecutive patients presenting with classic lacunar syndromes were studied. All had acute cranial CT to exclude primary intracerebral haemorrhage and these were subsequently assessed for the presence and extent of leukoaraiosis. All patients were genotyped for the ACE insertion/deletion polymorphism.

Results: There was a significant difference in the distribution of ACE genotype with the DD genotype occurring more often in patients with leukoaraiosis and the II and ID genotypes occurring more often among those in whom this was absent $\left(\chi^{2}=9.06, p=0.01\right)$. In a logistic regression model the ACE DD genotype remained as an independent predictor for the presence of leukoaraiosis $(p=0.02)$ in patients presenting with classic lacunar syndromes.

Conclusion: This study supports the hypothesis that there may be different types of small vessel disease in patients with classic lacunar syndromes and that the influence of the ACE DD genotype may be relevant in mediating the diffuse form of vessel injury.
\end{abstract}

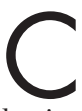
linical and pathological evidence suggests that patients with lacunar syndromes arising from occlusion of single cerebral small vessels may be a heterogenous group. The basis of this hypothesis stems from the original pathological studies of Fisher, ${ }^{1-3}$ who reported that serial sections of postmortem tissue showed two types of small vessel change. In specimens taken from selected patients with untreated hypertension widespread changes involving the vascular media which he termed lipohyalinosis were found. ${ }^{1}$ This affected predominantly the distal arteries less than $200 \mu \mathrm{m}$ in diameter and was associated with small, usually multiple, and asymptomatic ischaemic lacunes. In later case studies of patients with isolated, symptomatic lacunar infarction, intimal microatheromatosis at the origin of the deep perforating arteries was the most frequent underlying cause. ${ }^{2}$ After the introduction of CT, a group of patients who had hypointensity in the periventricular white matter (later termed leukoaraiosis) were identified. This radiological appearance, which is also thought to be a consequence of small vessel disease, ${ }^{4}$ seems to be present in some patients with lacunar syndromes but not others for reasons that are not yet fully explained.

Epidemiological evidence for the role of different underlying small vessel pathologies has been provided by Boiten et al. ${ }^{5}$ They found that patients presenting with lacunar syndromes fell into two groups: those with isolated symptomatic lacunar infarcts or those who also had multiple, usually asymptomatic, lacunar infarcts on imaging. Hypertension and leukoaraiosis were more likely to be encountered in the second group, supporting the existence of different underlying vascu- lopathies in the two phenotypes. Recent studies examining cerebral blood flow have been consistent with this notion of heterogeneity and suggested that endothelial dysfunction may play a particularly important part in the pathophysiology of certain patients with small vessel disease-that is, those with multiple lacunes or leukoaraiosis. ${ }^{67}$

Whereas genetic factors are thought to be important in cerebrovascular disease, ${ }^{89}$ except for rare familial disorders-for example, CADASIL ${ }^{10}$ or homocysteinuria ${ }^{11}$ where a single gene defect is thought to be responsible-the underlying candidate genes in most cases have yet to be implicated consistently. Given that certain risk factors such as hypertension ${ }^{5}$ and homocysteine concentrations ${ }^{12}$ may predispose to certain forms of vessel damage more than others, by analogy such phenotypic specificity may be applicable equally to genetic risk factors.

The gene encoding angiotensin converting enzyme (ACE) is thought to be an important candidate in cerebral small vessel disease because of the role of its peptide in hypertension, endothelial function, and the regulation of smooth muscle proliferation and tone. Two polymorphic alleles of the ACE gene can be identified within intron 16 depending on the presence of an insertion or deletion sequence (I or D). Allelic differences at this position have been reported to account for up to $47 \%$ of the variance in ACE concentrations with the

Abbreviations: $A C E$, angiotensin converting enzyme; I/D, insertion/deletion; BMI, basal metabolic index 


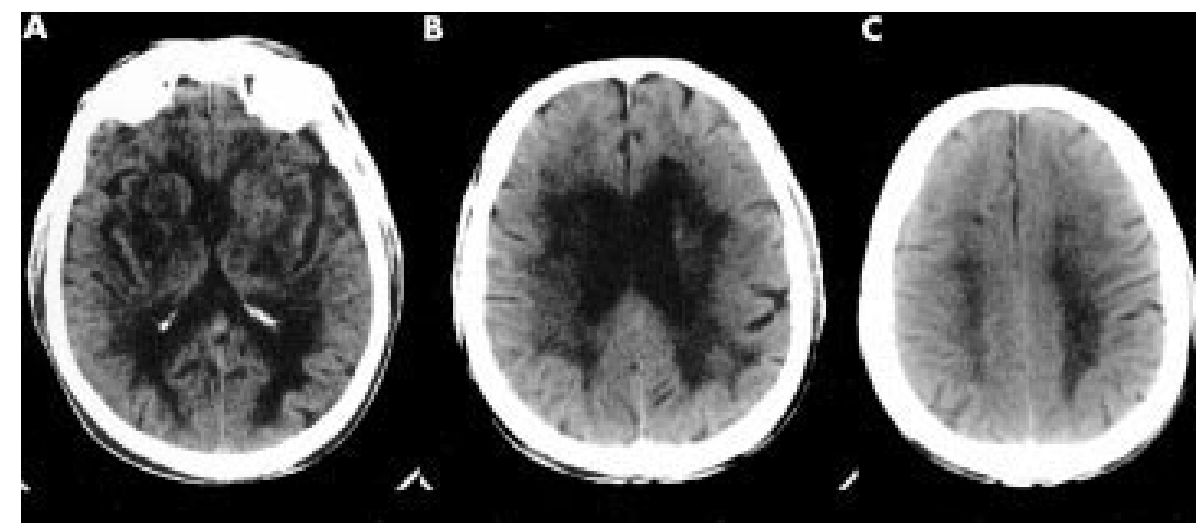

Figure 1 Brain CT sections show leukoaraiosis visible as low attenuation in the periventricular regions in a patient with a clinical lacunar stroke. (A) A lacune is also visible in the left ganglionic region. This case was scored as 4 according to the scale of van Swieten. ${ }^{20}$

highest concentrations typically found in those with the homozygous DD genotype. ${ }^{13}$ Association studies examining the lacunar stroke phenotype, have, however, been inconsistent, with conflicting reports concerning the importance of the DD genotype. ${ }^{14-17}$ One possible explanation is the existence of small vessel heterogeneity with the ACE genotype being more closely related to one of the specific subtypes. We examined this hypothesis in our study by determining whether an association could be found between the DD genotype and the presence or absence of leukoaraiosis in patients presenting with classic lacunar syndromes.

\section{MATERIALS AND METHODS \\ Study population}

Eighty four consecutive white patients who presented to one of four hospitals with an acute, classic lacunar syndrome as defined by the Oxfordshire Community Stroke Project Classification $^{18}$ were studied. The patients resided within the Leeds Family Health Services Authority and were recruited prospectively as part of a larger study of the influence of haemostatic and genetic risk factors in cerebrovascular disease. ${ }^{15}$ Informed consent was obtained according to a protocol approved by the hospital research ethics committee. Detailed clinical data including information on vascular risk factors were collected, together with physical findings. Hypertension was defined as a preadmission systolic blood pressure of at least $160 \mathrm{~mm} \mathrm{Hg}$ or a diastolic blood pressure of $95 \mathrm{~mm} \mathrm{Hg}$ or greater as recorded in the general practitioner's notes on two occasions. A history of ischaemic heart disease was assessed using the Rose questionnaire ${ }^{19}$ and with reference to ECGs and laboratory data. Body mass index (BMI) was defined as the weight $(\mathrm{kg})$ divided by the square of the height $(\mathrm{m})$. Current or previous smoking habits were also recorded.

Uncuffed venous blood samples were drawn from patients within 10 days of stroke onset for extraction of DNA.

\section{Scoring of leukoaraiosis and lacunes}

All enrolled patients had non-contrast cranial CT to exclude primary intracerebral haemorrhage and confirm a radiological lesion compatible with a lacunar event. The scans were subsequently made available to two independent radiologists for assessment of leukoaraiosis. The scans were interpreted according to a previously published protocol, ${ }^{20}$ using sections through (a) the choroid plexus, (b) sella media, and (c) centrum semiovale to record focal or diffuse hypointensity (fig 1). Periventricular hypointensity was scored out of two in the anterior and posterior white matter regions to give a total out of four. The number of lacunes, defined as sharply marginated low density lesions less than $15 \mathrm{~mm}(1.5 \mathrm{~cm})$ in maximum diameter, without mass effect and lying within the territory of a single perforating artery was also scored. In the event of a disagreement between the two radiologists the scans were arbitrated by a third assessor. Interpretation of scans was performed blind to the clinical and genotyping data.

\section{Laboratory techniques}

Genomic DNA was extracted from leucocytes isolated from 10 $\mathrm{ml}$ venous blood anticoagulated with $1.6 \mathrm{mg} / \mathrm{mL}$ EDTA using a detergent/salt exchange method as described previously. ${ }^{21}$ The ACE I/D polymorphism was detected by polymerase chain reaction according to the presence of the intron 16 specific insertion or deletion fragments, $490 \mathrm{~kb}$ and $190 \mathrm{~kb}$ respectively. The amplicons were separated on $2 \%$ agarose gels and visualised using ethidium bromide staining and ultraviolet light. Typing was performed blind to the clinical or radiological status of the patients. In instances where the DD genotype was identified this was verified using a second intron 16 specific amplication (5'-TTTGAGACGGAGTCTCG (TC- $\left.-3^{\prime}\right) .^{22}$

\section{Statistical methods}

The proportion of patients with lacunar infarct with and without leukoaraiosis were compared by $\chi^{2}$ testing. Continuous variables were analysed using the unpaired $t$ test. For BMI and cholesterol, values were log transformed, expressed as antilogged geometric means with 95\% confidence intervals (95\% CIs). The median number of lacunes scored in each group was compared using the Mann-Whitney $U$ test. Determinants of leukoaraiosis were studied in a logistic regression model with age, sex, BMI, hypertension, diabetes, number of lacunes, and ACE DD genotype as covariates. Statistical testing was performed using SPSS for Windows version 6.1 (SPSS).

\section{RESULTS}

Characteristics of the study population

Of the 84 patients with lacunar syndromes studied (table 1) there was no CT evidence of leukoaraiosis in $25(30 \%)$. In the remaining $59(70 \%)$, the grade of leukoaraiosis scored ranged from 1-4 and was fairly evenly distributed between the four grades of severity ( fig 2). The mean age of the patients with leukoaraiosis was greater than those without $(\mathrm{p}<0.0001)$. The male:female ratio was similar between the two groups and there was no significant difference in conventional cardiovascular risk factors including smoking history, ischaemic heart disease, hypertension, diabetes, and cholesterol concentrations. It was found that the patients with leukoaraiosis had a lower BMI than those without $(p=0.002)$. The median number of lacunes scored was also significantly greater in patients with leukoaraiosis $(p=0.01)$. 
Table 1 Characteristics of patients with and without leukoaraiosis

\begin{tabular}{llll}
\hline & With leukoaraiosis (+) & Without leukoaraiosis (-) & $\mathrm{p}$ Value \\
\hline Number & 59 & 25 & \\
Mean age (y) & $75(73-77)$ & $59(54-65)$ & $\mathrm{N} 0.0001$ \\
Sex (M/F) & $30 / 29$ & $14 / 11$ & $\mathrm{NS}$ \\
Diabetes & 8.5 & 24 & $\mathrm{NS}$ \\
Ever smoked & 44 & 49 & $\mathrm{NS}$ \\
Hypertension & 39 & 60 & $\mathrm{NS}$ \\
Ischaemic heart disease (\%) & 24 & 12 & 0.002 \\
BMI (kg/m & $23.4(22.2-24.7)$ & $28.2(25.8-30.8)$ & $\mathrm{NS}$ \\
Cholesterol (mmol/l) & $5.6(5.0-6.1)$ & $6.2(5.1-7.3)$ & 0.01 \\
Median lacunes scored (range) & $3(0-8)$ & $1(0-3)$ & \\
\hline \multirow{2}{*}{ Results are given as mean (95\% CI) unless stated otherwise. Values for BMl and cholesterol are expressed as } \\
antilogged geometric means (95\% Cl). Proportions are expressed as a percentage.
\end{tabular}

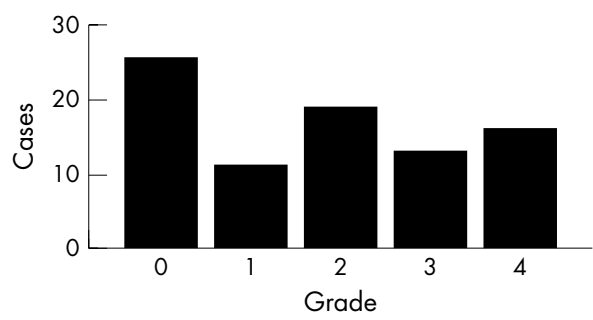

Figure 2 The frequency and grade of leukoaraiosis in 84 patients with clinical lacunar syndromes according to the scale of van Swieten et al..$^{20}$ (This is the sum of the scores allocated to the anterior and posterior white matter regions with the range of possible scores in each region being $0-2$.)

Table 2 Comparison of ACE genotype among the two groups

\begin{tabular}{lll}
\hline Genotype & Leukoaraiosis (+) & Leukoaraiosis (-) \\
\hline II & $10(16.9)$ & $8(32)$ \\
ID & $25(42.4)$ & $15(60)$ \\
DD & $24(40.7)$ & $2(8)$ \\
\hline
\end{tabular}

Figures are $\mathrm{No}(\%)$. There was a significant difference in the distribution of ACE genotype $\left(\chi^{2}=9.06\right.$, df $2, p=0.01$ between cases with and without leukoaraiosis.

\section{Genotype distribution in patients with and without leukoaraiosis}

The ACE I/D polymorphism genotype was available in all eighty four patients (table 2). There was a significant difference in distribution of the ACE genotype between the two groups $\left(\chi^{2}=9.06, \mathrm{df} 2, \mathrm{p}=0.01\right)$. On analysis of the $\chi^{2}$ table, it was apparent that these differences lay between patients with and without the DD genotype.

In a logistic regression model significant independent predictors for the presence of leukoaraiosis in the study population were age $(p=0.02)$, BMI $(p=0.01)$, the number of lacunes $(p=0.01)$, and possession of the DD genotype $(p=0.02)$. Separate logistic regression models were created to study possible interactions between the DD genotype and age or hypertension but failed to demonstrate any such relation.

\section{DISCUSSION}

The results from this study suggest that there is an association between ACE genotype and the presence of leukoaraiosis in patients presenting with non-haemorrhagic classic lacunar syndromes. Periventricular hypointensity is a non-specific radiological feature which may result from various causes such as demyelination, Alzheimer's disease, sarcoidosis, and vasculitis. ${ }^{23}$ In the clinical setting of lacunar stroke, however, it is accepted that the leukoaraiosis is ischaemic and most likely a consequence of small vessel disease. ${ }^{4}$ Evidence from selected pathological studies has suggested that patients with small vessel disease may represent a heterogenous group with ischaemia resulting from either localised atherosclerotic disease in the proximal segment of the deep perforating arteries, or more diffuse lipohyalinosis affecting the more distal segments. ${ }^{1-3}$ Clinical comparisons support the notion of distinguishing two types of vasculopathy, with hypertension, multiple lacunes, and leukoaraiosis being found more often in those suspected of having a diffuse vasculopathy. ${ }^{5}$ Consistent with these findings we found that patients with leukoaraiosis had more lacunes scored on CT. This would be compatible with a diffuse underlying disease process in this group. Analysis of the clinical characteristics of the two groups showed that the only traditional vascular risk factor which was significantly different between the two groups was age with the leukoaraiosis group being older. We were unable to show an association with hypertension, perhaps reflecting the limited size of our study and the high number of hypertensive patients encountered in both groups.

It is possible that additional factors such as genotypic variation could be responsible for modulating differences in the small vessel disease phenotype. The ACE gene was investigated in the light of previous studies which have suggested a functional role of the I/D polymorphism and the importance of ACE in vascular pathology, particularly endothelial function and hypertension. Two case-control studies have previously found an association between the DD genotype and lacunar stroke, ${ }^{14}{ }^{17}$ yet other reports have failed to replicate these findings. ${ }^{15}{ }^{16}$ One explanation for these differences could be the case mix of patients with and without leukoaraiosis as our study shows that the genotypic distribution differered between patients with the two subtypes. Additionally, we compared ACE genotype frequencies in our patients with ischaemic lacunar stroke with previously published control populations. ${ }^{14}{ }^{15}$ Whereas there were no major differences when the entire group was considered, analysis of the different subtypes showed that the DD genotype seemed to be overrepresented in patients with leukoaraisosis and underrepresented in those patients with isolated lacunar infarcts.

There are several possible explanations for the difference in genotypic distribution seen in patients with and without leukoaraisosis. Firstly, it could be due to a confounding effect of the ACE gene on longevity with enrichment of the DD genotype in elderly populations. ${ }^{24}$ However, using regression analysis we were unable to show a significant interaction between the DD genotype and age and the relation between the DD genotype and leukoaraiosis was independent. Secondly, the association may reflect the importance of the DD genotype in mediating the diffuse form of small vessel disease. The possible mechanisms underlying this association remain open to speculation but would seem to be independent of hypertension. It is possible that recently noted effects of the ACE genotype on endothelium ${ }^{25}$ may be involved as endothelial 
dysfunction may be particularly important in patients with leukoaraiosis. ${ }^{6} \mathrm{~A}$ third possibility is that the DD genotype protects against localised atherosclerotic disease, thereby favouring the development of diffuse disease in those who have a suitable risk factor profile.

The potential limitations of our study include the relatively few patients studied and the fact that patients had CT rather than MRI to evaluate leukoaraiosis. As MRI is more sensitive, it is possible that some patients with isolated lacunar infarcts may have had small amounts of leukoaraiosis and been incorrectly classified. However, the pathological significance of minor amounts of leukoaraiosis which may have been missed remains unclear although it is an important consideration for future studies.

In conclusion, our results are consistent with previous data suggesting that patients with ischaemic lacunar syndromes are a heterogenous phenotype and support the involvement of the ACE gene in mediating these differences. The presence of leukoaraiosis in patients with ischaemic lacunar syndromes may signify a distinct vascular injury process, a hypothesis that warrants further investigation in larger studies.

\section{ACKNOWLEDGEMENTS}

This work was kindly supported by the Stroke Association and the St James's Trust for Nervous System Diseases.

\section{Authors' affiliations}

A Hassan, A Lansbury, J M Bamford Department of Neurology, St James's University Hospital, Beckett Street, Leeds LS9 7TF, UK A Guthrie, J Spencer, C Craven, Department of Radiology, St James's University Hospital

A J Catto, P J Grant, Department of Molecular Vascular Medicine, Research School of Medicine, Leeds General Infirmary, Leeds LS1 3EX, UK

\section{REFERENCES}

1 Fisher CM. The arterial lesions underlying lacunes. Acta Neuropathol (Berl) 1968;12:1-15.

2 Fisher CM. Capsular infarcts: the underlying vascular lesions. Arch Neurol 1979;36:65-73.

3 Fisher CM. Lacunar strokes and infarcts: a review. Neurology 1982;32:871-6.

4 Hijdra A, Verbeeten B Jr, Verhulst JA. Relation of leukoaraiosis to lesion type in stroke patients. Stroke 1990;21:890-4.

5 Boiten J, Lodder J, Kessels F. Two clinically distinct lacunar infarct entities? A hypothesis. Stroke 1993;24:652-6.
6 Matsushita K, Kuriyama Y, Nagatsuka K, et al. Periventricular white matter lucency and cerebral blood flow autoregulation in hypertensive patients. Hypertension 1994;23:565-8.

7 Mochizuki Y, Oishi M, Takasu T. Cerebral blood flow in single and multiple lacunar infarctions. Stroke 1997;28: 1458-60.

8 Brass LM, Isaacsohn JL, Merikangas KR, et al. A study of twins and stroke. Stroke 1992;23:221-3

9 Carmelli D, DeCarli C, Swan GE, et al. Evidence for genetic variance in white matter hyperintensity volume in normal elderly male twins. Stroke 1998;29:1177-81.

10 Joutel A, Corpechot C, Ducros A, et al. Notch3 mutations in CADASIL, a hereditary adult-onset condition causing stroke and dementia. Nature 1996:383:707-10.

11 Kraus JP, Le K, Swaroop M, et al. Human cystathionine beta-synthase cDNA: sequence, alternative splicing and expression in cultured cells. Hum Mol Genet 1993;2:1633-8.

12 Fassbender K, Mielke O, Bertsch T, et al. Homocysteine in cerebral macroangiography and microangiopathy. Lancet 1999;353:1586-7.

13 Rigat B, Hubert C, Alhenc-Gelas F, et al. An insertion/deletion polymorphism in the angiotensin l-converting enzyme gene accounting for half the variance of serum enzyme levels. J Clin Invest 1990:86:1343-6.

14 Markus HS, Barley J, Lunt R, et al. Angiotensin-converting enzyme gene deletion polymorphism. A new risk factor for lacunar stroke but not carotid atheroma. Stroke 1995;26:1329-33.

15 Catto A, Carter AM, Barrett JH, et al. Angiotensin-converting enzyme insertion/deletion polymorphism and cerebrovascular disease. Stroke 1996;27:435-40

16 Pullicino P, Kwen PL, Greenberg S, et al. Angiotensin-converting enzyme gene and lacunar stroke. Stroke 1996;27:569-70.

17 Elbaz A, Mallet C, Cambien F, et al. on behalf of the GENIC investigators. Association between the ACE 4656 (CT) 2/3 polymorphism and plasma ACE levels with lacunar stroke in the GENIC study [abstract]. Cerebrovasc Dis 1998;8:44.

18 Bamford J, Sandercock P, Jones L, et al. The natural history of lacunar infarction: the Oxfordshire community stroke project. Stroke 1987; 18:545-51.

19 Rose G, McCartney P, Reid DD. Self administration of a questionnaire on chest pain and intermittent claudication. Br J Prev Soc Med 1977;31:42-8.

20 van Swieten JC, Hijdra A, Koudstaal PJ, et al. Grading white matter lesions on CT and MRI: a simple scale. J Neurol Neurosurg Psychiatry 1990:53:1080-3.

21 Gustincich S, Manfioletti G, Del Sal G, et al. A fast method for high-quality genomic DNA extraction from whole human blood. Biotechniques 1930;11:298-300.

22 Shanmugam V, Sell KW, Saha BK. Mistyping ACE heterozygotes. Genome Res 1993;3:120-1.

23 Brown MM. Leukoaraiosis In: Donnan GA, Norving B, Bamford JM, et al, eds. Lacunar and other subcortical infarctions. Oxford, Oxford University Press, 1995: 180-98.

24 Schachter F, Faure-Delanef L, Guenot F, et al. Genetic associations with human longevity at the APOE and ACE loci. Nat Genet 1994;6:29-32.

25 Butler R, Morris AD, Burchell B, et al. DD angiotensin-converting enzyme gene polymorphism is associated with endothelial dysfunction in normal humans. Hypertension 1999;33:1164-11. 